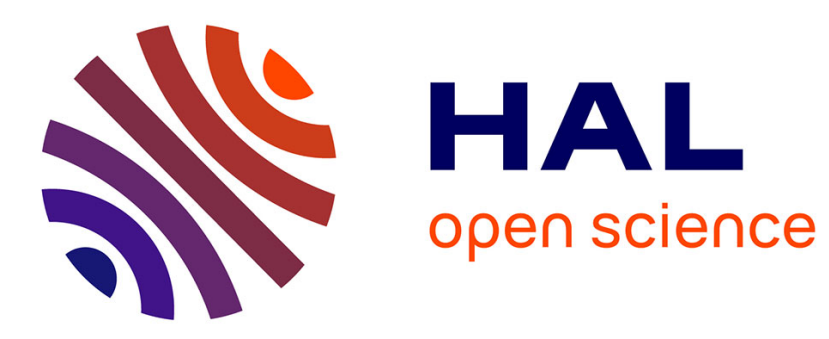

\title{
Siegfried face au chant de l'Oiseau
}

Nicolas Rabain

\section{To cite this version:}

Nicolas Rabain. Siegfried face au chant de l'Oiseau: Effets des interactions précoces et quête des origines à l'adolescence. Topique - Revue freudienne, 2014, Écouter Wagner, 128, pp.97-107. 10.3917/top.128.0097 . hal-01411203

\section{HAL Id: hal-01411203 https://hal.science/hal-01411203}

Submitted on 9 Dec 2016

HAL is a multi-disciplinary open access archive for the deposit and dissemination of scientific research documents, whether they are published or not. The documents may come from teaching and research institutions in France or abroad, or from public or private research centers.
L'archive ouverte pluridisciplinaire HAL, est destinée au dépôt et à la diffusion de documents scientifiques de niveau recherche, publiés ou non, émanant des établissements d'enseignement et de recherche français ou étrangers, des laboratoires publics ou privés. 


\section{Siegfried face au chant de l'Oiseau}

Effets des interactions précoces et quête des origines à l'adolescence

En hommage à Ruth Lebovici,

« L'interaction mère/nourrisson apparaît ainsi comme le prototype primitif de toutes les formes ultérieures d'échanges. Dans cette « conversation » entre la mère et le bébé, les mots et les phrases sont remplacés, souvent chez la mère, toujours chez le jeune nourrisson, par des messages extra-verbaux : gestes, vocalisations, sourires, etc. »

(1) (S. Lebovici, 1983)

Seul sous un tilleul,bercépar les murmures de la forêt, Siegfried rêve. Soudain, un hautbois, une flûte et une clarinette interprètent un thème pentatonique qui éveille la curiosité du jeune homme. Ces trois instruments à vent de la famille des bois entonnent le chant de l'Oiseau. Comment comprendre ce langage d'emblée impénétrable ? S'agirait-il d'une énigme ? L'Oiseau serait-il un messager ? Que viendrait-il annoncer à Siegfried juste avant son combat avec l'effroyable dragon Fafner?

Après la mort du monstre, ce même oiseau réapparaît. Siegfried comprend alors son chant provenant cette fois-ci d'une voix de soprano. Tantôt interprété par une femme, tantôt par un enfant, l'Oiseau éclaire le destin du héros pendant une grande partie du deuxième acte de ce drame lyrique.

Historiquement, on sait que Wagner a provisoirement abandonné la composition de Siegfried après avoir achevé le premier acte et seulement ébauché le deuxième. Quels obstacles aurait rencontrésl'artiste au moment où son héros s'apprête à tuer Fafner et Mime avant de prendre femme ? Douze années de latence auront été nécessaires au musicien avant qu'il ne puisse mener à terme la partition concernant la fin de la jeunesse de son héros.

Dans cet article, nous proposerons de considérerSiegfried- tel qu'il apparaît dans l'opéra éponyme de Wagner - comme une allégorie de l'adolescence. A cette occasion, nous revisiteronscertaines notions psychanalytiquesliées au remaniement des imagos parentales et à la quête identitaire à cet âge de la vie.Si le chant de l'Oiseau renseigne le jeune homme sur les dangers qu'il encourt et la mission héroïque qu'il doit accomplir, nous dégagerons de ces passages deux hypothèses.

Dans un premier temps, notre attention sera portée sur la question des interactions précoces. Le chant de l'Oiseau renverrait-ild'une certaine manière aux échanges et au corpsà-corps de la mère et du bébé ?Dans cette perspective, Siegfried, face à l'Oiseau, serait confronté au lien précoce qu'il a entretenu avec son substitut maternel.

Puis, nousconvoquerons dans un second temps le concept de narrativité, et plus précisément l'acquisition d'une compétence auto-narrative à l'adolescence.Cette référence théorique nous conduira à nous demander si l'Oiseau n'inciterait pas Siegfried à mettre en récit ses propres origines.

Avant d'aborder ces points, penchons-nousau préalable sur la place des oiseaux dans la mythologie nordique. Les quatre livrets du Ring montrent combien Wagner était imprégné de l'Edda poétique, ensemble de textesécrits dans une langue scandinave médiévale appelée levieux norrois. Datant du XIII ${ }^{\text {ème }}$ siècle, ce recueil de poèmes islandais est appelé le Codex 
Regius. Il constitue l'une des premières traces écrites de cette mythologie transmise jusqu'alors oralement (2) (S. Sturluson, 1991).

Dans ces poèmes, Siegfried apparaît sous le nom de Sigurdr et Mime, celui de Reginn. Après la mise à mort du dragon nommé ici Fáfnir, ce n'est pas l'Oiseau mais sept mésanges qui apparaissent. La première recommande à Sigurdr de faire rôtir le cœur du dragon mort et de le manger. Quant aux six autres mésanges, elles conseillent d'abord à Sigurdr de tuer Reginn ; puis, elles indiquent au héros le chemin qui le conduira à « une vierge, de beaucoup la plus belle. » (3) (R. Boyer, 1992).

Dans l'œuvre de Wagner, cesdeuxmeurtres successifsont lieu à proximitéd'une caverne située dans une épaisse forêt entre les roches et les crevasses. Le géant Fafner y sommeille également sous la forme d'un dragon terrifiant afin de protéger l'Anneau des Nibelungen. Siegfried atteint la grotte aux côtés de Mime, nain qui l'a élevé depuis sa naissance. Car Sieglinde, la mère du héros, enceinte de son propre frère jumeau Siegmund, a expiré à peine après avoir donné naissance au fruit de ses amours incestueuses. Elle avait confié au gnome son fils ainsi que deux morceaux de l'épée Notung. Mime, pourtant forgeron, n'est jamais parvenu à ressouder les deux bouts de l'arme. Aussi a-t-il élevé Siegfried dans le but de réparer Notung, l'épée de Siegmund ayant été détruite par Wotan lors d'un dernier combat. Sans avoir réparé au préalable l'arme de son père, Siegfried ne pourraitvaincre le dragon et ravir l'Anneau(4)(Wagner, 1876).

\section{De la reviviscence de l'archaïque à l'adolescence}

\section{Les murmures de la forêt}

Arrivé à la caverne, Siegfried chasse Mime de sa vue, convaincu que le nain ne peut être son véritable père. Musicalement, une transition progressive s'opère entre le thème de $L a$ forge - thème saccadé, répétitif et tenant sur trois notes -et l'idylle des Murmures de la forêtmélodieuse, aérienne et apaisante. Ce passage au cours duquel Siegfried rêve renvoie au Prélude de L'or $d u$ Rhin à un bémol près : cette idylle est en mi majeur(5)(Lavignac, 1897), soit un demi ton au-dessus des cent trente sept mesuresinaugurales, caractérisées par une succession de quintes, d'octaves et de tierces en mi bémol majeur. De la même manière que l'auditeur se trouve plongé dans « un temps sacré » (6) (M. Pazdro, 1988) au début duRing, il est à nouveauinvité à méditer sur les origines de l'existence.

Assoupi dans la forêt, le jeune homme paraît ici renvoyé aux origines du monde tout autant qu'à sa propre naissance. A cette occasion, certains souvenirs et certaines sensations anciennes semblent refaire surface au moment même où émergent des questions identitaires. D'où vient-il ? Qui sont ses parents ? Pourquoi sa mère est-elle morte en couche ?Musicalement, ce passage oscille entre exaltation lorsqu'il évoque la mère comme premier objet d'amour, et profonde afflictionquand il s'agit de sa mère à jamais perdue. Ce dualisme entre exaltation et affliction, présent dans le livret, est également restitué par le génie musical du compositeur. Par ce procédé, Wagner permet à son public de ressentir avec finesse les éprouvés émotionnels de l'adolescent passant de l'enthousiasme d'être libre au désarroi d'être seul. Cette humeur inconstante est restituée par une alternance d'accords majeurs et mineurs qui donnent à entendre et à percevoir combienlaideur et beauté se côtoient sans nuance à cet âge de la vie.De la quiétude à la détresse, il n'y a qu'un pas.

Ainsi, Siegfried, faceà lui-même, est troublé par cette sensation de liberté nouvelle. Confronté à ses souvenirs et à son monde interne, il passe brutalement du désarroi d'avoir 
perdu prématurément sa mère,à des moments d'attendrissement au cours desquels il évoque une mère idéalisée qu'il n'a jamais connue.

Dans ce passage, défenses maniaques, élans dépressifs et humeur révoltée sont autant d'éléments qui témoignent de la labilité émotionnelle de l'adolescent face à un passé qui tend à refaire surface. Au cœur de cette scène s'inaugure un retour du refoulé, ou plus précisément une levée du refoulement concernant l'amnésie infantile.

Après une longue période de latence dans une sombre forêt où la sexualité se serait endormie - et après douze ans d'interruption pour Wagner dans la composition de l'œuvre ce retour du refoulé est initiéconjointement à l'évocation du regard de la mère. C'est par le truchement de ce regard - imaginé en ce qui concerne Siegfried dont la mère est morte en couche, mais vraisemblablement reconstruit à partir de l'expérience infantile du compositeur - que le jeune homme est renvoyé à son identité. Le regard des femmes aimées paraît icicentral. A cet égard, on retrouvera dans l'acte suivant le même champ lexical pour qualifier le regard de Brünnhilde :étincelantpour la mère - «Ses yeux étincelants de clarté » („Ihr hellschimmernde Augen") - et éclatantpour l'épouse - «Je vois clairement l'éclat de ton regard » („Deines Auges Leuchten; seh' ich licht“). Le regard des femmes éclaire la forêt obscure où le héros a grandi loin de toute présence féminine. Cet éclairage du regard maternel permettrait alors àSiegfried de «se voir lui-même » comme le font envisager les travaux de Winnicottconcernant le rôle de miroir du visage de la mère(7) (D. Winnicott, 1971).

Nous sommes ici au cœur du processus adolescent où les questions de la labilité émotionnelle et de la sensorialité sont au premier plan. A un niveau plus œdipien, Siegfried est également traversé par un profond sentiment de culpabilité. Il pense avoir tué sa mèrepar le simple fait d'être né. Ce fantasme de matricide fait échoà une problématique centrale chez nombre d'adolescents : comment s'éloigner de sa mère sans la tuer et sans mourir soi-même ? Ce passage illustre à quel point la distinction entre éloignement et perteest floue chez Siegfried. A cet égard, rappelons ici que Wagner a commencé la composition du Ring par La mort de Siegfried (Siegfrieds Tod) en 1848, année où sa propre mère s'est éteinte. La disparition du fils paraît ici peu différenciée du décès de la mère.

A ce stade de notre propos, nous pouvons introduire une distinction entre différentes imagos maternelles desquelles l'adolescent devra se départir au cours de son parcours initiatique : mère séductrice et mère dévoratrice, notamment. Tuer la mère archaïque, ou encore mettre à distance la mère séductrice, sont autant de défis pour Siegfried avant sa rencontre avec Brünnhilde.

Le fantasme de matricide de Siegfried semble s'accompagner d'un clivage entre deux types de représentations maternelles.Dans une perspective kleinienne, on observe l'évocation d'une mère morte idéalisée s'opposant à des imagos maternelles dangereuses, incarnées à la fois par certains traits du dragon et de Mime.

Ainsi, le doublemeurtre du géant et du nain pourrait également être lu non seulement comme le meurtre du père sur un plan œdipien, mais également comme celui de la mauvaise mère dans une autre perspective d'analyse centrée sur le matériel préœdipien. Si à première vue, Fafner apparaît comme une imago paternelle forte et dangereuse, il n'en reste pas moins envisageable que son aspect terrifiant convoque également des fantasmes archaïques.Plus particulièrement, ceux de dévoration et d'engloutissement qu'il est possible d'attribuer à une représentation de la mauvaise mère au sens où l'entend Mélanie Klein (8) (M. Klein, 19211945).

De la même manière, le personnage de Mime condense à lui seul des représentations paternelles et maternelles. A ce sujet, le livret est explicite puisque dès le début de l'opéra, le 
nain se dit être «à la fois le père et la mère »[de Siegfried] („Ich bin dir Vater und Mutter zugleich“.) (9) (R. Wagner, 1876)Ainsi, Mime incarnerait la condensation de deux imagos repoussantes: autant celle d'un père manipulateur et dangereux que celle d'une mère archaïque capable de concocter des breuvages empoisonnés pour celui qu'il nomme «1'adolescent exécrable. »(,Der schmähliche Knabe“)(10)(Wagner, 1876).

Ainsi, Siegfried est répugné par celui qui l'a élevé et rasséréné à l'idée d'avoir eu des parents plus nobles.La question de la nostalgie est ici centrale, celle de l'époque où la mère et par extension les parents - était tout pour l'enfant. La surestimation des premières années de vie trahit, comme Freud l'a développé, la difficulté à se détacher de ses parents (11) (S. Freud, 1909).Cette difficulté est envisagée chez l'adolescent qui pleure la mère perdue de son enfance juste avant que n'arrive l'Oiseau.

\section{Le chant de l'Oiseau}

Sur un thème pentatonique joué par un trio d'instruments à vent, l'Oiseau apparait à Siegfried ; le sens de son chant est d'emblée inaccessible mais intrigue toutefois le héros. Cevolatile provient du haut des cieux où règne la lumière et où résident les âmes des défunts. Laseule présence de l'Oiseau au langage d'abord incompréhensible donne suffisamment de courage au héros pour occire l'effroyable monstre.Dans cette scène de meurtre, l'adolescent surmonte certes ses propres angoisses sans difficulté apparente, mais soulignons qu'il n'est plus seul. L'Oiseau au regard bienveillant reste à proximité du héros comme pour le protéger.

Interrogeons-nous maintenantsur l'accueil que Siegfried fait du chant de celui qu'il pense n'être qu'un simple animal.Sans en comprendre le sens, l'adolescent fait face à une langue « impénétrable [...] opaque, hiéroglyphique »(12) (A. Satgé, 1993). Il coupe alors un roseau à l'aide de son glaive et confectionne un pipeau pour tenter d'entrer en lien avec l'Oiseau par mimétisme. Ce procédé de mimétisme rappelle les travaux initiés par ColwynTrevarthen qui font écho à des recherches neuroscientifiques sur les neurones miroirs mis en jeu dans la communication infra-verbale entre la mère et le bébé. Ils corroborent le fait que cette fonction miroirest nécessaire pour que le bébé puisse entrer en contact avec son propre monde affectif, voire son propre monde représentatif(13) (G. Rizzolatti, C. Sinigaglia, 2008).

Revenons à Siegfried qui, dans un premier temps, ne parvient pas à reproduire le leitmotiv de l'Oiseau: «Cela sonne faux ! (...) Petit oiseau, il me semble que je reste bien sot: tes leçons ne sont guère aisées ! »(,Das tönt nicht recht (...) Vöglein, mich dünkt; ich bleibe dumm: von dir lernt sich's nicht leicht".) (14) (R. Wagner, 1876) Le pipeau semble symboliser l'impuissance de l'enfant qui ne comprend pas encore le langage de la sexualité comme le souligne $\mathrm{S}$. Ferenczi dans ses travaux sur la confusion de langue entre les adultes et l'enfant (15)(S. Ferenczi, 1932).

Apprivoiser l'Oiseau reviendrait-il à se familiariser avec le langage de la sexualité adulte ? Dans ce cas-là, l'Oiseau prendrait une figure incitatrice et initiatrice. Une dimension excitante propre à la figure maternelle semble ici mise en scène. A cet égard, nous pourrions faire l'hypothèse que le chant de l'Oiseau est emprunt de signifiants énigmatiques au sens où Jean Laplanche les a conceptualisés dans sa théorie de la séduction généralisée(16) (J. Laplanche, 1987).Dans cette perspective, ne pourrait-on pas entendre le chant de l'Oiseau comme un message énigmatique issu de l'inconscient parental ? Ce chant véhiculeraitalors « un sens à lui-même ignoré » qu'il reviendrait à Siegfried de traduire dans l'après-coup. 
Quel serait le devenir de ces messages adressés jadis à l'enfant lorsque son appareil psychique a traversé la période de latence ?Pour Isée Bernateau, «l'adolescence viendrait[...] rejouer dans un cadre nouveau des partitions déjà connues, de telle sorte que des harmonies ou des disharmonies, inouïes jusqu'alors, peuvent se faire entendre. »(17) (I. Bernateau, 2008). Cette métaphore musicale invite àentrevoir l'adolescence comme une période de prédilection pour sinon parvenir à traduire, du moins tenter de déchiffrer ces signifiants énigmatiques.

Dans un second temps,Siegfried se saisit de son cor en argent après avoir jeté au loin le roseau, tel un outil de l'enfance devenu inutile. Il joue avec assurance son propre leitmotiv, « la virtuosité du solo du cor rachetant les fausses notes du roseau. »(18) (A. Satgé, 1993). Le dragon s'éveille alors. Pour le jeune homme, le moment est venu de se débarrasser d'un monstre terrorisant dont la denture effrayantele laisse paradoxalement de marbre. Siegfried est le héros qui méconnaît la peur, vertu qui lui permet d'être le seul à pouvoir abattre le monstre.

On peut envisager ici un lien avec la reviviscence d'angoisses archaïques, rééditées au moment de la puberté comme l'ont souligné les travaux de Philippe Gutton. D'après l'auteur, il ne s'agit toutefois pas d'une répétition à l'identique, mais au contraire d'une " ouverture possible au remaniement »(19) (Ph. Gutton, 1991).En effet, la maturation progressive de l'appareil psychique a permis de rendre désormais inoffensifs les scénarios jadis terrifiants.

Ainsi, c'est à proximité d'une représentation du ventre maternel - la caverne - que l'adolescent triomphe de ses angoisses archaïques pour renaître au monde en héros.De la dépendance à autrui propre à l'adolescence, Siegfried s'éloigne. En outre,il s'affranchit de ses craintes d'enfant, se rapprochant parallèlement de la possibilité d'un changement d'objet d'amour ; car bientôt, il passera d'un investissement exclusif de sa mère à celui d'une amante.

Avant d'y parvenir, une compétence nouvelle naît progressivement en lui du fait de l'intérêt soudain qu'il porte à son histoire et à ses origines. La mise en récit de sa vie constitue le second élément sur lequel Siegfried s'appuiera pour prendre son envol.

\section{Vers l'acquisition d'une compétence auto-narrative}

Si Siegfried semble converser avec la mère idéalisée de l'enfance lorsqu'il s'adresse à l'Oiseau, il n'en reste pas moins pertinent d'y voir également un dialogue intérieur avec luimême. En somme, face aux gazouillis de l'Oiseau, on peut envisager l'hypothèse selon laquelle Siegfried se parle. Il converserait avec l'enfant qu'il a été. On peut y entrevoir une tentative de réappropriation subjective de sa propre histoire.

À cet égard, avant derendre l'âme et après avoir repris une apparence humaine, Fafner demande à Siegfried qui il est. Mais le héros ne connaît rien de sa propre identité. Avant de trépasser, le moribond transmet à Siegfried ce qu'il sait de l'épopée du Ring et de la malédiction de l'Anneau. Siegfried en est bouleversé : «Eclaire-moi encore sur mes origines, ô sauvage, puisque tu sembles plein de sagesse au moment de mourir. »(,Woher ich stamme, rate mir noch; weise ja scheinst du, Wilder, im Sterben“.) (20) (R. Wagner, 1876)

Si Fafner évoque entre autre une imago paternelle dominatrice et effrayante lorsqu'il a la formed'un dragon, sa transformation en vieil homme proche du trépas semble émouvoir et intéresser Siegfried. La puissance du fils s'instaure ici face à un père affaibli comme le prédisait Mime dès le premier acte : «A la force juvénile de Siegfried, Fafner pourrait bien succomber. » („Siegfrieds kindischer Kraft, erläge wohl Fafners Leib“.) (21) (R. Wagner, 1876) 
En récupérant son glaive après que Fafner a rendu son dernier souffle, Siegfried se brûle la main en touchant au sang du défunt. Il porte alors les doigts à sa bouche pour les rafraîchir et comprend soudain le chant de l'Oiseau qui l'éclaire sur les missions qu'il devra accomplir. La brûlure de Siegfried dans l'opéra de Wagner est un reliquat d'une scène explicite dans l'Edda poétique où Sigurdr se brûle la main avec le sang du dragon dont l'Oiseau lui a recommandé de faire rôtir le cœur pour le manger. Dévorer le cœur du dragon plutôt que de se laisser dévorer par lui, tel est le premier exploit du jeune homme. Quant à la brûlure, marque d'un rituel initiatique éludé par Wagner, symboliserait-elle le prix à payer pour avoir accès au langage de la sexualité ?

L'écoulement de sang brûlant semble ici renvoyer l'adolescent à plusieurséléments comme celui de sa naissance et celui de l'effroi face à la blessure d'une figure paternellerendant l'âme. On n'est pas loin des craintes de Parsifal face à la blessured'Amfortas (22) (R. Wagner, 1886).La nécessité de porter secours à un père en bravant soi-même un grand nombre de dangers est un point que Parsifal et Siegfried partagent. En d'autres termes, devant l'imminence de la mort du père, on observe que l'identification à ses traits jugés valeureux et nobles serait comme facilitée.

Revenons à l'Oiseau qui, par sa «voix prophétique»(23) (B. Lussato, 2005),vient protéger Siegfried en lui révélant tout ce qu'il ne peut voir et qui lui est toutefois impératif de savoir. Une voix de soprano annonce trois messages dans une langue à laquelle le jeune homme a désormais accès. Un des principauxmandats qui a été déposé à son endroit est maintenant formulé : ildevra d'abord récupérer l'Anneau caché dans la caverne.A cet égard, ce mandat lui vient autant de Mime, son substitut paternel, que de Wotan, son grand-père. Il s'agit à la fois d'un mandat inter- et transgénérationneldont la distinction prend une place importante dans les travaux de Serge Lebovici (24) (S. Lebovici, 1998).On observe ici combien ce défi lancé par le père d'adoption est pris dans des enjeux de conflits œedipiens inhibant sa réalisation; au contraire, lorsquela même demande émane d'un désir grandpaternel, le héros récupère presqu'aussitôt ce dont les Dieux du Walhalla ont été dépossédés.

Rapidement après l'agonie de Fafner, Mime réapparaît et compte récupérer le trésor lui-même après avoir empoisonné Siegfried. Grâce aux oracles énoncés par l'Oiseau, le meurtre du géant est donc suivi de peu par le meurtre du nain. Ainsi libéré, mais toutefois esseulé,Siegfried se lamente de n'avoir ni père ni mère. L'Oiseau fait une troisième apparition et lui apprend qu'une femme l'attend, endormie sur un rocher entouré de flammes : c'est la Walkyrie nommée Brünnhilde - fille que son grand-père Wotan a eu avec Erda - lamère des origines, «DieUrmutter ».

Les trois messages de l'Oiseau ont ainsi fait passer Siegfried de la niaiserie adolescente du premier acte au héros du troisième acte, prêt à braver les flammes pour réveiller la Walkyrie et enfin apprendre à travers elle ce qu'est la peur.

Dans cet article, nous avons défendu l'idée que l'Oiseauamènerait Siegfried à dialoguertour-à-touravec sa mèreet avec l'enfant qu'il a été.Dans ces deux cas, cet animal renverrait à un passé auquel illui est nécessaire de se confronter afin de s'approprier subjectivement sa propre histoire et de pouvoir accomplir la mission dont il a été investi.

Si l'Oiseau est central dans le deuxième acte de Siegfried, il concerne également le dernier acte du Crépuscule des Dieux(25) (R. Wagner, 1876). Chaque fois que l'Oiseau apparait, il est question pour le héros de se remémorer des scènes de son passé.Au cours des premiers échanges avec l'Oiseau, Siegfriedse rappelle sa mère défunte, comme nous l'avons vu précédemment. En revanche, avant de mourir, le thème de l'Oiseau permet au héros de se souvenir de Brünnhilde qu'un philtre avait fait disparaître de sa mémoire. 
Cette dernière apparition de l'Oiseau renvoie à nouveaule héros à son attachement aux femmes aimées : à Brünnhilde certes, mais également à la mère de son enfance. Ainsi, les images de ces deux femmes, peu différenciées l'une de l'autre lorsque Siegfried rencontre Brünnhilde, se confondent à nouveau au moment de la mort du héros.

Nicolas Rabain,

Psychologue clinicien, Premier Secteur de Psychiatrie infanto-juvénile du Val-de-Marne, ATER à l'UFR d'Études Psychanalytiques de l'Université Paris Diderot - Paris 7

Rattaché au CRPMS

@: nrabain@hotmail.com 


\title{
Résumé
}

Ce travail propose un dialogue entre une œuvre de Wagner et la psychanalyse. D'un côté, Siegfried - en tant qu'allégorie de l'adolescence - face au chant énigmatique de l'Oiseau ; de l'autre, certaines notions psychanalytiques issues de la clinique des adolescents.

Le livret et la musique du deuxième acte de Siegfried sont abordés ici sous l'angle de deux perspectives : d'une part, la reviviscence du lien précoce à l'objet primaire ainsi que la résurgence des angoisses archaïques à l'adolescence; et d'autre part, les modalités d'acquisition d'une compétence auto-narrative.

Mots-clés

Siegfried - Wagner - Adolescence - Interactions précoces - Archaïque - Narrativité

\begin{abstract}
This paper presents a dialogue between a work by Wagner and psychoanalysis. On one side, Siegfried - as an allegory for adolescence -enchantedby the enigmatic song of the Bird; on the other, some psychoanalytic concepts stemming from clinical work with adolescents.

The libretto and the music of the second act of Siegfried are discussed here from two perspectives: first, the revival of the early bond to the primary object and the resurgence of archaic anxieties; and second, the modalities of acquiring an auto-narrative competence.
\end{abstract}

\section{Keywords}

Siegfried - Wagner - Adolescence - Early Interactions - Archaic - Narrativity 


\section{Notes}

1. S. Lebovici, S. Stoléru, Le nourrisson, sa mère et le psychanalyste - Les interactions précoces, (1983), Bayard, 2003, p. 96.

2. S. Sturluson, L'Edda-Récits de mythologie nordique, Gallimard, 1991, p. 119-127.

3. R. Boyer, «Le meurtre de Fáfnir » in L'Edda poétique, Fayard, 1992, p. 310-322.

4. R. Wagner, Siegfried, (1876), trad. de J. d'Arièges, Flammarion Bilingue, 1971.

5. A. Lavignac, Le voyage artistique à Bayreuth, (1897), Librairie Delagrave, 1900, p. 429-430.

6. M. Pazdro, Guide des opéras de Wagner, Fayard, 1988, p.708.

7. D. W. Winnicott, «Le Rôle de miroir de la mère et de la famille dans le développement de l'enfant »in Jeu et réalité, (1971), Gallimard, 1975, p. 153-162.

8. M. Klein, Essais de psychanalyse,(1921-1945), Payot, 1967.

9. R. Wagner, Siegfried, (1876), trad. de J. d'Arièges, Flammarion Bilingue, 1971, p. 6465.

10. R. Wagner, Siegfried, (1876), trad. de J. d'Arièges, Flammarion Bilingue, 1971, p. 4445.

11. S. Freud, «Le roman familial des névrosés » in Névrose, psychose et perversion, (1909), PUF, 1973, p. 157-160.

12. A. Satgé, «Les circuits du savoir » in Siegfried, L’Avant Scène Opéra, 1993, p. 10.

13. G. Rizzolatti et C. Sinigaglia, Les neurones miroirs, Odile Jacob, 2008.

14. R. Wagner, Siegfried, (1876), trad. de J. d'Arièges, Flammarion Bilingue, 1971, p. 174-175.

15. S. Ferenczi, «Confusion de langue entre les adultes et l'enfant», (1932), in CEuvres complètes - Tome IV, (1927-1933), Payot, 1982, p.125-135.

16. J. Laplanche, Nouveaux fondements pour la psychanalyse, (1987), - Quadrige, 2008.

17. I. Bernateau, « La séparation, un concept pour penser les relations précoces et leur réaménagement à l'adolescence » in La psychiatrie de l'enfant, Vol. 51, 2008, p. 440.

18. A. Satgé, «Les circuits du savoir » in Siegfried, L'Avant Scène Opéra, 1993, p. 10.

19. Ph. Gutton, Le pubertaire, Editions PUF - Le fil rouge, Paris, 1991, 322 pp.

20. R. Wagner, Siegfried, (1876), trad. de J. d'Arièges, Flammarion Bilingue, 1971, p. 183.

21. R. Wagner, Siegfried, (1876), trad. de J. d'Arièges, Flammarion Bilingue, 1971, p. 47.

22. R. Wagner, Parsifal, (1882), trad. de M. Beaufils, Flammarion Bilingue, 1979.

23. B. Lussato, Voyage au cour du Ring, Fayard, 2005, p. 134-135.

24. S. Lebovici, L'arbre de vie - Eléments de la psychopathologie du bébé, (1998),Erès, 1998.

25. R. Wagner, Le crépuscule des Dieux, (1876), trad. de J. d'Arièges - Flammarion Bilingue, 1972. 


\section{Bibliographie}

I. Bernateau, «La séparation, un concept pour penser les relations précoces et leur réaménagement à l'adolescence » in La psychiatrie de l'enfant, Vol. 51, 2008, pp. 425-455.

R. Boyer, L'Edda poétique, Fayard, 1992.

S. Ferenczi, « Confusion de langue entre les adultes et l'enfant », (1932), in Euvres complètes - Tome IV,(1927-1933), Payot, 1982, p.125-135.

S. Freud, «Le roman familial des névrosés » in Névrose, psychose et perversion, (1909), PUF, 1973, p. 157-160.

Ph. Gutton, Le pubertaire, Editions PUF - Le fil rouge, Paris, 1991, 322 pp.

E. Kestemberg, «L'identité et l'identification chez les adolescents », (1962),in L'adolescence à vif, PUF, 1999 : p. 7-96.

M. Klein, Essais de psychanalyse,(1921-1945),Payot, 1967.

J. Laplanche, Nouveaux fondements pour la psychanalyse, (1987), - Quadrige, 2008.

A. Lavignac, Le voyage artistique à Bayreuth, (1897), Librairie Delagrave, 1900.

S. Lebovici, L'arbre de vie - Eléments de la psychopathologie du bébé, Erès, 1998.

S. Lebovici, S. Stoléru, Le nourrisson, sa mère et le psychanalyste - Les interactions précoces, (1983), Bayard, 2003.

B. Lussato, Voyage au cour du Ring, Fayard, 2005.

M. Pazdro, Guide des opéras de Wagner, Fayard, 1988.

G. Rizzolatti et C. Sinigaglia, Les neurones miroirs, Odile Jacob, 2008.

A. Satgé, «Les circuits du savoir » in Siegfried, L’Avant Scène Opéra, 1993, p. 4 -15.

S. Sturluson, L'Edda - Récits de mythologie nordique, Gallimard, 1991.

R. Wagner, Siegfried, (1876), trad. de J. d'Arièges,Flammarion Bilingue, 1971.

R. Wagner, Le crépuscule des Dieux,(1876), trad. de J. d'Arièges - Flammarion Bilingue, 1972.

R. Wagner, Parsifal, (1882), trad. de M. Beaufils, Flammarion Bilingue, 1979.

D. W. Winnicott, «Le Rôle de miroir de la mère et de la famille dans le développement de l'enfant »in Jeu et réalité, (1971), Gallimard, 1975, p.153-162. 


\section{Plan de construction}

Titre

Epigraphe

\section{Introduction}

Propos introductifs

Annonce du plan et des perspectives de l'article

Remise en contexte du thème

Première partie : De la reviviscence de l'archaïque à l'adolescence Première sous-partie : Les murmures de la forêt

Seconde sous-partie : Le chant de l'Oiseau

Seconde partie : Vers l'acquisition d'une compétence auto-narrative

\section{Conclusion}

Auteur

Résumé et mots-clés en français puis en anglais

Notes

Bibliographie 